\title{
Revisão
}

\section{Uso da laserterapia no tratamento de pacientes com paralisia de Bell: revisão crítica da literatura}

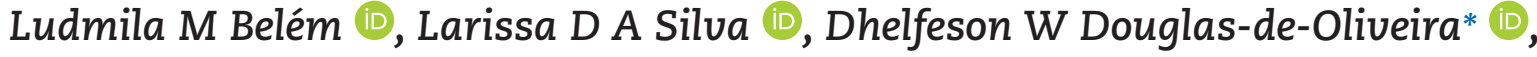 Patrícia F Gonçalves (D), Olga D Flecha}

Departamento de Odontologia, Faculdade de Ciências Biológicas e da Saúde, Universidade Federal dos Vales do Jequitinhonha e Mucuri, Diamantina, Minas Gerais, Brasil.

INFORMAÇÃO SOBRE O ARTIGO

\section{Historial do artigo:}

Recebido a 17 de abril de 2020

Aceite a 9 de maio de 2021

On-line a 8 de junho de 2021

\section{Palauras-chave:}

Paralisia de Bell

Reabilitação

Terapia a laser

\begin{abstract}
R E S U M O
O objetivo foi identificar a evidência acerca da efetividade da laserterapia no tratamento de paralisia de Bell. Foram incluídos ensaios clínicos indexados na Pubmed, SciELO, Lilacs e na literatura cinzenta, sem limites de data. Foram incluídos 5 estudos, dos quais dois tinham nível de evidência I e três nível de evidência III. A laserterapia de baixa intensidade foi o tratamento mais frequente e as terapias associadas foram exercícios e massagens faciais, terapia magnética, bloqueio do gânglio estrelado e biofeedback/eletromiografia. Concluiu-se que há poucos estudos de boa qualidade metodológica e níveis de evidência para garantir a efetividade da laserterapia no tratamento de paralisia de Bell. Contudo, os resultados sugerem a redução ou a resolução das sequelas da paralisia de Bell dos pacientes tratados com laser, em combinação ou não com outras terapias. (Rev Port Estomatol Med Dent Cir Maxilofac. 2021;62(2):81-86)
\end{abstract}

(C) 2021 Sociedade Portuguesa de Estomatologia e Medicina Dentária. Publicado por SPEMD. Este é um artigo Open Access sob uma licença CC BY-NC-ND

(http://creativecommons.org/licenses/by-nc-nd/4.0/).

\footnotetext{
* Autor correspondente.

Correio eletrónico: dhelfeson@hotmail.com (Dhelfeson W Douglas-de-Oliveira).

http://doi.org/10.24873/j.rpemd.2021.06.832

1646-2890/@ 2021 Sociedade Portuguesa de Estomatologia e Medicina Dentária. Published by SPEMD.

This is an open access article under the CC BY-NC-ND license (http://creativecommons.org/licenses/by-nc-nd/4.0/).
} 


\title{
Use of laser therapy in the treatment of patients with Bell's palsy: a critical review of the literature
}

\author{
A B S T R A C T
}

Keywords:

Bell palsy

Rehabilitation

Laser therapy
This review aimed to identify the evidence about the effectiveness of laser therapy in the treatment of Bell's palsy. It included clinical trials indexed in the Pubmed, SciELO, and Lilacs databases, and from the gray literature, with no date limit. Five studies were included, of which two had a level of evidence I, and three a level of evidence III. Low-level laser therapy was the most frequent treatment, and the combined therapies were facial exercises with massages, magnetic therapy, star-crossed ganglion block, and biofeedback/electromyography. In conclusion, few studies of good methodological quality and levels of evidence have been published to ensure the effectiveness of laser therapy in the treatment of Bell's palsy. However, the results suggest the sequels of Bell's palsy are reduced or resolved in patients treated with laser, associated or not with other therapies. (Rev Port Estomatol Med Dent Cir Maxilofac. 2021;62(2):81-86)

(c) 2021 Sociedade Portuguesa de Estomatologia e Medicina Dentária. Published by SPEMD. This is an open access article under the CC BY-NC-ND license (http://creativecommons.org/licenses/by-nc-nd/4.0/).

\section{Introdução}

A Paralisia de Bell (PB) é também conhecida por paralisia idiopática facial ou paralisia facial periférica e afeta $07 .^{\circ}$ nervo craniano, de maneira repentina, podendo causar paralisia total ou parcial dos músculos da face. Dentre os danos causados, podem-se citar os distúrbios de paladar, de salivação, lacrimejamento, hiperacusia e perda da sensibilidade no local. Estima-se que as mulheres sejam as mais acometidas e que alguns fatores de risco, como: hipertensão arterial, Diabetes Mellitus, gravidez e puerpério e a infecção pelo vírus Herpes simplex (tipo I), possam ser considerados para a PB, embora a sua etiologia ainda não seja confirmada. ${ }^{1}$

Estima-se que a PB corresponda de $60 \%$ a $75 \%$ de todas as causas de paralisias faciais existentes e, apesar de a maioria ter um bom prognóstico, cerca de $20 \%$ a $30 \%$ dos pacientes, que não conseguem se recuperar totalmente, apresentam problemas psicológicos motivados pela assimetria facial, lesões oculares, pelas dores na face e pelas sincinesias, que são as contraturas involuntárias dos músculos faciais. ${ }^{2}$

Várias são as terapias que podem ser escolhidas para o tratamento da PB, entre elas, podem ser citadas o tratamento farmacológico, a acupuntura e eletro acupuntura, os exercícios terapêuticos/reabilitação neuromuscular e o LASER de alta ou baixa intensidade. ${ }^{3}$ Diante de todas as modalidades terapêuticas mencionadas, o LASER, em conjunto com as outras terapias existentes, é considerado eficaz para a reabilitação dos pacientes. ${ }^{3}$

O LASER é capaz de obter um crescimento na amplitude dos potenciais de ação (função nervosa estimulada) e possui capacidade de acelerar a regeneração de algumas estruturas nervosas. Uma possível hipótese para o mecanismo pela qual a luz do LASER possa estimular a reinervação de tecidos é sua penetração nos axónios ou nas células de Schwann adjacentes, induzindo o metabolismo do tecido nervoso danificado a produzir proteínas associadas com o crescimento do nervo, ou lançando um fator de trofismo. Além disso, a laserterapia mostra-se efetiva na minimização da administração de fármacos nos pacientes com PB. ${ }^{4}$

Este estudo tem por objetivo identificar evidência na literatura científica acerca da efetividade da laserterapia no tratamento de pacientes com Paralisia de Bell, através de uma revisão crítica.

\section{Material e Métodos}

As pesquisas para o desenvolvimento deste estudo foram realizadas nas bases de dados Pubmed, SciELO, Lilacs e na literatura cinzenta (Google Scholar, Periódicos Capes) com critérios de inclusão e exclusão pré-definidos, com as palavras-chave obtidas no DECS e no MeSH, isoladas ou combinadas, em inglês e português: "paralisia de Bell, LASER, laserterapia, paralisia facial idiopática”.

Foram incluídos estudos do tipo ensaios clínicos, randomizados ou não, em qualquer idioma, sem definição de data, em pacientes com $\mathrm{PB}$, independente de raça, género e idade. A intervenção de interesse foi o uso do LASER como terapia, sem restrições quanto ao tipo, modelo e protocolo em comparação a outras terapias e suas eficácias na redução dos sinais e sintomas da PB.

Uma análise detalhada e completa dos artigos de escolha para o estudo foi efetuada e vários aspetos foram levados em conta, para realizar a avaliação final. Estes aspetos foram: autor/ ano, desenho do estudo, tamanho da amostra, objetivos, critérios de inclusão, intervenções, LASER e protocolos e conclusão.

Para avaliar a qualidade dos ensaios clínicos, criou-se um quadro de acordo com o Consolidated Standards of Reporting Trials (CONSORT), ${ }^{5}$ adaptado de S. He et al., ${ }^{6}$ com critérios estabelecidos ${ }^{7}$ para qualificar a metodologia e classificar os níveis de evidência, sendo eles: cálculo amostral, randomização, ocultação da alocação, mascaramento e perdas no seguimento. ${ }^{8}$ Quando o critério fosse considerado adequado (reportado 
e explicado) seria estabelecida a classificação A, se fosse apenas mencionado sem explicações, era classificado como B e quando não fosse nem mencionado, receberia letra C. ${ }^{6,7}$

A pesquisa, leitura dos artigos, seleção e avaliação crítica dos estudos selecionados foi feita por duas avaliadoras (LMB e LDAS), previamente treinadas e calibradas.

O ensaio clínico que preenchesse completamente os critérios, ou quatro destes, seria avaliado como nível I de evidência. Se cumprisse parcialmente os critérios (máximo duas avaliações C), seria considerado como nível II e caso tivesse seguido dois ou menos critérios, teria avaliação III nos níveis de evidência. ${ }^{6,7}$

Para a avaliação final, cada artigo foi classificado de acordo com os critérios estabelecidos. A seguir foram separados e avaliados de acordo com cada item e classificados conforme os níveis de evidência obtidos.

\section{Resultados}

No início das pesquisas, foram encontrados 11 artigos. Após a aplicação dos critérios de inclusão, 5 foram separados para a leitura integral. ${ }^{9-13}$ Foram selecionados 3 estudos em inglês, 1 artigo em espanhol e 1 em português. A Figura 1 exibe um fluxograma, em que mostra a identificação, o rastreamento e a inclusão dos estudos, para a realização da revisão crítica.

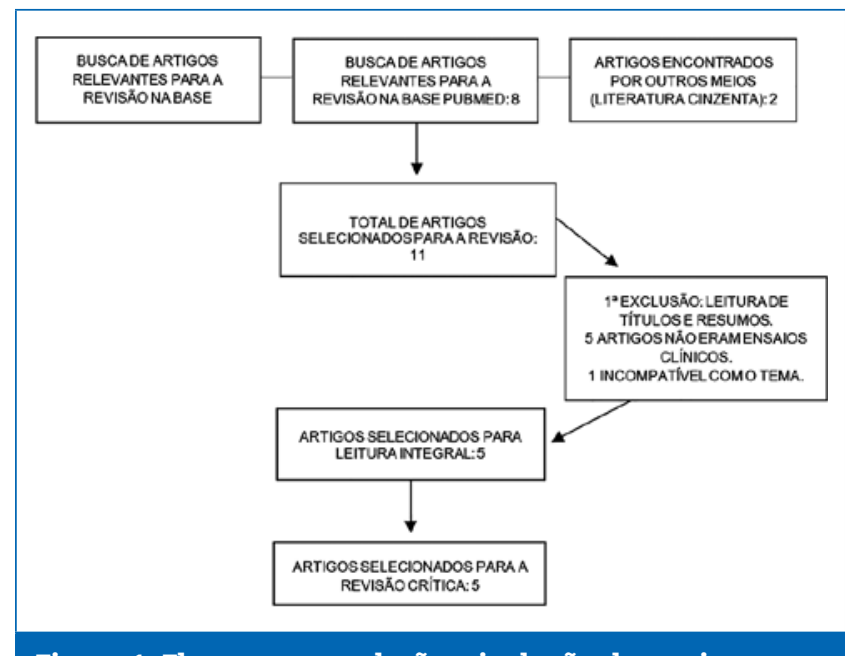

Figura 1. Fluxograma: seleção e inclusão dos artigos

Os estudos incluídos nesta revisão tiveram níveis de evidência I ou III, de acordo com os critérios estabelecidos para avaliar a qualidade de ensaios clínicos randomizados. Os cinco artigos que preencheram total ou parcialmente os critérios de inclusão eram ensaios clínicos, dois com ocultação de alocação, dois com mascaramento e três mencionaram perdas no seguimento. Dois estudos explicaram como foi feito o cálculo da amostra. Apenas dois ensaios clínicos foram considerados como nível de evidência I e três foram avaliados como III (Tabela 1). ${ }^{6,7,14}$ Estudos que foram classificados como nível de evidência I, são considerados como com baixo risco de viés e são os melhores em qualidade metodológica. Aqueles qualificados como III podem ser portadores de alto risco de viés, por não relatarem informações suficientes contidas em um estudo de boa qualidade.

A Tabela 2 mostra uma sinopse dos 5 estudos selecionados e, de acordo com os níveis de evidência, suas classificações. É importante salientar a enorme variedade na qualidade metodológica, a discrepância de intervenções realizadas e a heterogeneidade das variáveis encontradas.

\section{Discussão}

O uso do LASER como terapia na paralisia de Bell tem sido tomado em consideração nos últimos tempos pela sua facilidade, porque é indolor, não invasivo, poder abranger diferentes perfis de pacientes e por ser um meio mais favorável para aqueles que não podem usar medicamentações do tipo corticosteroides, como pacientes com hipertensão arterial e Diabetes Mellitus. ${ }^{15}$

Apresenta resultados na regeneração nervosa, em défices neurosensoriais e neuromotores, ${ }^{16}$ e pode ser eficaz na regeneração do nervo periférico ferido e também para mitigara degeneração dos neurónios. ${ }^{17-19}$ Os efeitos podem ser locais e sistémicos, descritos como anti-inflamatórios, anti-edematosos e anti-dor penetrando profundamente nos tecidos, e é, portanto, uma terapia a considerar no tratamento da PB. ${ }^{20}$

A literatura sobre o assunto é escassa. Por conseguinte, este estudo de revisão foi limitado, visto que poucos estudos foram encontrados e a maioria dos incluídos foi considerada de baixa qualidade metodológica e alto risco de viés, de parcialidade.

Os estudos ${ }^{9,10}$ que obtiveram o nível de evidência I, seguiram quase todos os critérios estabelecidos, tornando-os mais fiáveis e com o máximo nível de evidência, sendo considerados, portanto, ensaios clínicos de qualidade. Um ensaio clínico randomizado é considerado padrão ouro de qualidade, na avaliação das intervenções de saúde e é considerado como o segundo nível de evidência para a tomada de decisões. ${ }^{8}$

Embora os autores ${ }^{9}$ tenham atendido à maioria dos critérios, as perdas de seguimento são mencionadas, mas não foi explicado como elas foram tratadas estatisticamente. As qualidades metodológicas de um ensaio clínico podem ser prejudicadas por ausências, retiradas e perdas dos participantes, o que pode anular a equivalência inicial dos grupos controlo e experimental. ${ }^{8,21}$

Alayat et. al. ${ }^{9}$ fizeram o mascaramento de modo duplo-cego (paciente e avaliador não conheciam a intervenção), tornando-o mais fiável, em relação a outros que eram simples cego ${ }^{10}$ (só o examinador não conhecia a intervenção) ou não tinham cegamento. ${ }^{11-13}$ Ensaios que não sejam duplo-cego podem produzir estimativas mais elevadas nos resultados dos tratamentos. ${ }^{8}$ O cegamento dos pacientes e dos envolvidos na aplicação das intervenções, quando possível, evita viés e também protege contra diferenças na utilização de placebos. ${ }^{8}$

Mascaramento (ou cegamento) não deve ser confundido com ocultação da alocação, da designação do paciente para as intervenções. A randomização depende de dois processos: geração de uma sequência de atribuição que é impossível prever e a ocultação dessa sequência até que a intervenção ocorra. Ela é apropriada se os pacientes e investigadores envolvidos não puderem prever a designação. A ocultação visa prevenir o 
Tabela 1. Sinopse dos estudos selecionados

\begin{tabular}{|c|c|c|c|c|c|c|c|c|}
\hline $\begin{array}{l}\text { Autores } \\
\text { e Ano }\end{array}$ & $\begin{array}{c}\text { Desenho } \\
\text { dos estudos }\end{array}$ & $\begin{array}{l}\text { Tamanho } \\
\text { amostral }\end{array}$ & Objetivos & $\begin{array}{l}\text { Critérios de } \\
\text { inclusão }\end{array}$ & Intervenções & LASER e protocolos & Conclusões & $\mathrm{NE}$ \\
\hline $\begin{array}{l}\text { Alayat } \\
\text { et al. }{ }^{9} \\
2014\end{array}$ & $\begin{array}{l}\text { Ensaio clínico } \\
\text { randomizado } \\
\text { duplo-cego } \\
\text { placebo } \\
\text { controlado }\end{array}$ & $\begin{array}{l}48 \text { pacientes; } \\
\text { média } \\
\text { de idade: } \\
43 \pm 9,8 \text { anos }\end{array}$ & $\begin{array}{l}\text { Comparar LASER } \\
\text { de alta e baixa } \\
\text { intensidade } \mathrm{x} \\
\text { exercícios no } \\
\text { tratamento da PB }\end{array}$ & $\begin{array}{l}\text { Pacientes que } \\
\text { tiveram PB } \\
\text { unilateral do lado } \\
\text { esquerdo ou direito }\end{array}$ & $\begin{array}{l}\text { Massagem facial e } \\
\text { exercícios nos } 3 \\
\text { grupos: dois deles } \\
\text { com o uso dos } \\
\text { LASER de alta e } \\
\text { baixa intensidade } \\
\text { associados }\end{array}$ & $\begin{array}{l}\text { Baixa Intensidade: } \\
\text { Infravermelho } 830 \mathrm{~nm} \\
-100 \mathrm{nW} 10 \mathrm{~J} / \mathrm{cm}^{2} \\
-8 \text { pontos } \\
2 \text { min } 5 \mathrm{~s} \text { por ponto } \\
18 \text { sessões } \\
\text { Alta Intensidade: } \\
\text { Infravermelho } 1,064 \mathrm{~nm} \\
-3 \mathrm{~kW} 10 \mathrm{~J} / \mathrm{cm}^{2} \\
7 \mathrm{~s} \text { por ponto } \\
18 \text { sessões }\end{array}$ & $\begin{array}{l}\text { LASER de Alta e } \\
\text { Baixa Intensidade } \\
\text { são eficazes } \\
\text { Alta Intensidade } \\
\text { foi o mais eficaz }\end{array}$ & I \\
\hline $\begin{array}{l}\text { Ordahan e } \\
\text { Karahan }^{10} \\
2017\end{array}$ & $\begin{array}{l}\text { Ensaio clínico } \\
\text { randomizado }\end{array}$ & $\begin{array}{l}46 \text { pacientes; } \\
\text { média } \\
\text { de idade: } \\
41 \pm 9,7 \text { anos }\end{array}$ & $\begin{array}{l}\text { Papel do LASER de } \\
\text { baixa intensidade } \\
\text { associado a } \\
\text { exercícios de } \\
\text { Flexibilização } \\
\text { Cerebral em } \\
\text { pacientes com PB }\end{array}$ & $\begin{array}{l}\text { Pacientes com PB } \\
\text { unilateral, } \\
\text { admitidos no } \\
\text { Hospital de } \\
\text { Pesquisa e Ensino } \\
\text { de Konya }\end{array}$ & $\begin{array}{l}\text { Laserterapia de } \\
\text { baixa intensidade } \\
\text { em associação a } \\
\text { exercícios de } \\
\text { Flexibilização } \\
\text { Cerebral }\end{array}$ & $\begin{array}{l}\text { Baixa Intensidade } \\
\text { Infravermelho } 830 \mathrm{~nm} \\
-100 \mathrm{Mw} 10 \mathrm{~J} / \mathrm{cm}^{2} \\
-8 \text { pontos } \\
2 \text { min por ponto } \\
3 \mathrm{x} \text { por semana } \\
6 \text { semanas }\end{array}$ & $\begin{array}{l}\text { Laserterapia de } \\
\text { baixa intensidade } \\
\text { combinada } \\
\text { com exercícios } \\
\text { = melhorias } \\
\text { significativas }\end{array}$ & I \\
\hline $\begin{array}{l}\text { Montero } \\
\text { et al. }{ }^{11} \\
2015\end{array}$ & $\begin{array}{l}\text { Ensaio clínico } \\
\text { randomizado }\end{array}$ & $\begin{array}{l}88 \text { pacientes; } \\
\text { idades entre } \\
25 \text { a } 34 \text { anos }\end{array}$ & $\begin{array}{l}\text { Comparar efeitos da } \\
\text { terapia a LASER e } \\
\text { Terapia Magnética, } \\
\text { avaliação clínica e } \\
\text { funcional }\end{array}$ & Não mencionados & $\begin{array}{l}\text { Grupo controle } \\
\text { recebeu terapia } \\
\text { magnética e grupo } \\
\text { de estudo recebeu } \\
\text { laserterapia }\end{array}$ & $\begin{array}{l}\text { Baixa Intensidade } \\
3 \text { min } \\
-3 \text { pontos } \\
10 \text { sessões }\end{array}$ & $\begin{array}{l}\text { Grupo tratado com } \\
\text { LASER obteve } \\
\text { resposta mais } \\
\text { rápida e eficaz e } \\
\text { sem complicações }\end{array}$ & III \\
\hline $\begin{array}{l}\text { Murakami } \\
\text { et al. }{ }^{12} \\
1993\end{array}$ & Ensaio clínico & $\begin{array}{l}52 \text { pacientes; } \\
\text { média } \\
\text { de idade: } \\
\text { 42,34 anos }\end{array}$ & $\begin{array}{l}\text { Comparar o } \\
\text { tratamento de PB } \\
\text { com a laserterapia } \\
\text { de baixa intensidade } \\
\text { e bloqueio do Stellate } \\
\text { Ganglion }\end{array}$ & Não mencionados & $\begin{array}{l}\text { Três grupos: grupo } \\
\text { de LASER; grupo } \\
\text { com bloqueio de } \\
\text { SG e um grupo } \\
\text { com ambas. }\end{array}$ & $\begin{array}{l}\text { Baixa Intensidade } \\
\text { Infravermelho } 830 \mathrm{~nm} \\
-60 \mathrm{~mW} \\
10 \mathrm{seg} \text { por ponto } \\
\text { Tempo máximo } \\
\text { de } 5 \text { a } 10 \text { minutos }\end{array}$ & $\begin{array}{l}\text { LASER produziu } \\
\text { recuperação inicial } \\
\text { mais rápida e } \\
\text { escores finais de } \\
\text { paralisia pouco } \\
\text { melhores em } \\
\text { relação ao } \\
\text { bloqueio de SG }\end{array}$ & III \\
\hline $\begin{array}{l}\text { Januário } \\
\text { et al. }{ }^{13} \\
2012\end{array}$ & Ensaio clínico & $\begin{array}{l}22 \text { pacientes; } \\
\text { idades entre } \\
43 \text { e } 79 \text { anos }\end{array}$ & $\begin{array}{l}\text { Analisar efeitos } \\
\text { do biofeedback } \\
\text { por EMG e do LASER } \\
\text { de baixa intensidade } \\
\text { na função física e } \\
\text { bem-estar social em } \\
\text { pacientes com PB }\end{array}$ & $\begin{array}{l}\text { Indicação para } \\
\text { fisioterapia, com } \\
\text { mínimo de } 3 \text { meses } \\
\text { após lesão, } \\
\text { disfunção muscular } \\
\text { facial a partir } \\
\text { do grau II ao VI } \\
\text { segundo a escala de } \\
\text { House-Brackmann }\end{array}$ & $\begin{array}{l}\text { Grupo tratado } \\
\text { com LASER } \\
\text { e grupo tratado } \\
\text { com BFB/EMG }\end{array}$ & $\begin{array}{l}\text { Baixa Intensidade } \\
\text { Infravermelho } 904 \mathrm{~nm} \\
-25 \mathrm{~W} 15 \mathrm{~J} / \mathrm{cm}^{2} \\
-20 \mathrm{seg} \\
38 \text { pontos } \\
16 \text { sessões }\end{array}$ & $\begin{array}{l}\text { Ambos } \\
\text { favoreceram a } \\
\text { melhora da função } \\
\text { física e bem-estar } \\
\text { social dos } \\
\text { pacientes, porém o } \\
\text { BFB/EMG } \\
\text { mostrou-se mais } \\
\text { eficaz }\end{array}$ & III \\
\hline
\end{tabular}

Critérios adaptados de Nascimento et al., ${ }^{7}$ S. He et al. ${ }^{6}$ e Nogueira et al. ${ }^{14}$

Abreviaturas: BFB - Biofeedback; SG - Stellate Ganglion; PB - Paralisia de Bell; EMG - Eletromiografia; NE - Nível de Evidência

Tabela 2. Avaliação metodológica dos artigos selecionados

\begin{tabular}{|c|c|c|c|c|c|c|}
\hline Autor/ano & $\begin{array}{c}\text { Cálculo } \\
\text { amostral }\end{array}$ & Randomização & $\begin{array}{c}\text { Ocultação } \\
\text { da Alocação }\end{array}$ & Mascaramento & $\begin{array}{l}\text { Perdas no } \\
\text { segmento }\end{array}$ & NE \\
\hline Alayat et al. ${ }^{9}, 2014$ & $\mathrm{SIM}=\mathrm{A}$ & $\mathrm{SIM}=\mathrm{A}$ (computador) & $\mathrm{SIM}=\mathrm{A}$ & SIM=A (Duplo Cego) & $\mathrm{SIM}=\mathrm{B}$ & I \\
\hline Ordahan e Karahan ${ }^{10}, 2017$ & $\mathrm{SIM}=\mathrm{A}$ & SIM=A (computador) & $\mathrm{SIM}=\mathrm{A}$ & SIM=A (Simples Cego) & $\mathrm{SP}=\mathrm{A}$ & I \\
\hline Montero et al. ${ }^{11}, 2015$ & $\mathrm{NM}=\mathrm{C}$ & $\mathrm{SIM}=\mathrm{B}$ (Sem esclarecer) & $\mathrm{NM}=\mathrm{C}$ & $\mathrm{NM}=\mathrm{C}$ & $\mathrm{NM}=\mathrm{C}$ & III \\
\hline Murakami et al. ${ }^{12}, 1993$ & $\mathrm{NM}=\mathrm{C}$ & $\mathrm{NM}=\mathrm{C}$ & $\mathrm{NM}=\mathrm{C}$ & $\mathrm{NM}=\mathrm{C}$ & $\mathrm{NM}=\mathrm{C}$ & III \\
\hline Januário et al. ${ }^{13}, 2012$ & $\mathrm{NM}=\mathrm{C}$ & $\mathrm{NM}=\mathrm{C}$ & $\mathrm{NM}=\mathrm{C}$ & $\mathrm{NM}=\mathrm{C}$ & $\mathrm{SP}=\mathrm{A}$ & III \\
\hline
\end{tabular}

Nascimento et al. ${ }^{7}$ 
viés de seleção, protege a sequência de designação antes e até a alocação, e pode sempre ser realizada com sucesso. Por outro lado, o mascaramento procura evitar viés de determinação, controla o viés do observador e protege a sequência depois da alocação, o que nem sempre pode ser feito. ${ }^{8}$

Três estudos ${ }^{11-13}$ foram avaliados como nível de evidência III, com baixa qualidade metodológica e grande risco de viés. $\mathrm{O}$ estudo de Montero et. al. ${ }^{11}$ cumpriu apenas um critério na qualificação da metodologia - a randomização - mas não houve qualquer esclarecimento sobre como foi feita. Murakami et. al. ${ }^{12}$ e Januário et. al. ${ }^{13}$ sequer mencionaram sobre o procedimento. Randomização significa que cada paciente tem chance igual à dos outros participantes para receber qualquer tratamento, mas o tratamento a ser dado não pode ser previsto, portanto, ela controla o viés de seleção. ${ }^{8}$

Um estudo ${ }^{12}$ não mencionou nenhum dos itens estabelecidos, tendo sido então atribuído critério C em todos eles e o outro, ${ }^{13}$ também classificado como como nível de evidência III, informou não ter tido perdas no seguimento $(\mathrm{SP}=\mathrm{A})$, mas não cumpriu os outros itens.

É importante salientar que o estudo de Murakami et al. ${ }^{12}$ foi publicado em 1993, enquanto os outros ${ }^{9,10,11,13}$ foram entre 2012 e 2017, o que pode ser levado em conta, uma vez que naquela altura os estudos sobre o assunto eram escassos, fazendo com que o trabalho tivesse menos evidência científica e a rigidez metodológica não era um requisito com as exigências de hoje.

Um ponto forte a considerar nesta revisão é a pesquisa minuciosa em três das principais bases de dados disponíveis, a categorização de itens importantes para um bom nível de evidência dos ensaios clínicos encontrados e a sua avaliação crítica.

As limitações encontradas são, principalmente, na metodologia. A comparação entre os estudos torna-se difícil, devido à grande heterogeneidade dos procedimentos utilizados. Alguns $^{9,10}$ comparam a eficácia do LASER e de exercícios faciais, porém, o estudo de Alayat et al. ${ }^{9}$ compara o LASER de alta e baixa intensidade e exercícios e o estudo de Ordahan e Karahan ${ }^{10}$ apenas LASER de baixa intensidade e exercícios. Esses dois estudos $^{9,10}$ são os que mais se assemelham, uma vez que nos outros, ${ }^{11-13}$ as intervenções que são comparadas ao LASER são distintas (BioFeedback/Eletromiografia, ${ }^{13}$ bloqueio de Stellate Ganglion ${ }^{12}$ e terapia magnética ${ }^{11}$ ). Além disso, os modelos de LASER usados são variados. Dois estudos ${ }^{9,13}$ utilizaram o LASER diodo de Arseneto de Gálio (GaAs), mas de diferentes marcas. Ordahan e Karahan ${ }^{10}$ usaram o LASER Diodo de Gálio-Alumínio-Arseneto (GaAlAs). Outros, ${ }^{11,12}$ não mencionaram essa informação. Todos os LASER usaram o tipo infravermelho e eram de baixa intensidade, exceto $\mathrm{um}^{9}$ que também utilizou o LASER de alta intensidade, que foi de Neodímio (Nd).

Os autores ${ }^{9-12}$ concluem que a laserterapia é mais eficaz que as demais intervenções mencionadas, porém, Januário et al. ${ }^{13}$ concluíram que Biofeedback/Eletromiografia (BFB/EMG) obteve melhora estatisticamente mais rápida, quando comparada à laserterapia de baixa intensidade. Alayat et al. ${ }^{9}$ consideraram a laserterapia de alta intensidade a mais indicada, quando comparada com a laserterapia de baixa intensidade. $\mathrm{O}$ estudo realizado por Januário et al. ${ }^{13}$ mostra que tanto a laserterapia de baixa intensidade, quanto o BFB/EMG restauram a função física e o bem-estar social dos pacientes, no entanto, o segundo apresentou-se mais eficaz, mas é importante interpretar esse resultado com cautela, uma vez que ele tem baixa qualidade metodológica e os resultados podem ser tendenciosos se não houver rigor metodológico. ${ }^{8}$

Todos os estudos usaram LASER de baixa intensidade, com exceção do estudo ${ }^{9}$ que também utilizou o LASER de alta intensidade e o LASER infravermelho foi o mais frequente..$^{9-13} \mathrm{O}$ ensaio de Montero et al. ${ }^{11}$ não tem informações sobre o tipo do LASER, o que dificulta a comparação com os outros estudos e torna-se outra limitação nesta revisão. Os estudos utilizaram protocolos diferentes para os LASER, sendo distintos os valores de comprimento de onda, potência, frequência, energia, tempo de exposição e número de sessões. Os pontos que receberam as terapias foram vários, incluindo, no estudo ${ }^{11}$ pontos de acupuntura (estômago, vesícula biliar e bexiga) sendo utilizados.

As fases da doença em que as intervenções começaram também foram distintas. Nos estudos de boa qualidade metodológica, ${ }^{9,10}$ os autores referem que as intervenções começaram na fase subaguda da doença (3 a 5 dias após o declínio do quadro agudo). Em Januário et al., ${ }^{13}$ o tratamento começou na fase crónica da paralisia facial periférica (3 meses após o surgimento da lesão), o que sugere que o uso do LASER pode ser mais eficaz na fase subaguda da doença, uma vez que este estudo não obteve resultados significativos com a laserterapia, como relatam outros autores. ${ }^{9,10}$ Montero et al. ${ }^{11} \mathrm{e}$ Murakami et al. ${ }^{12}$ não mencionaram quando as intervenções ocorreram.

Houve várias escalas de avaliação do grau de recuperação facial. Em alguns estudos ${ }^{9,11,13}$ foi utilizada a escala House Brackmann. Ela é usada para avaliar o controlo evolutivo da paralisia facial e é considerada a escala mais aceite em todo o mundo, devido à sua facilidade de utilização e sensibilidade clínica. ${ }^{9}$ Esta escala foi aplicada aos pacientes antes e depois dos tratamentos, para comparações de possível melhoria. No estudo de Ordahan e Karahan, ${ }^{10}$ a escala de escolha foi a IDE (Índice de Incapacidade Facial), para avaliar a melhoria facial antes e depois da terapia ser realizada. No estudo de Murakami et al. ${ }^{12}$ a Escala Facial Nerve Research Group of Japan foi usada para avaliar o controlo evolutivo. Escalas para avaliar o índice de função física (IFF) e o índice de bem-estar social (IBES) dos pacientes foram utilizadas no estudo por Januário et al. ${ }^{13}$

Considera-se necessário que sejam realizados mais estudos sobre o uso da laserterapia no tratamento da PB, com qualidade metodológica adequada para fornecer evidência na literatura científica e fundamentos sobre o assunto. $O$ esclarecimento sobre o assunto torna-se importante uma vez que os pacientes poderiam procurar esta terapia com mais frequência e os profissionais se interessariam em realizar formação e estudos na área.

\section{Conclusões}

Embora existam poucos estudos de boa qualidade metodológica para garantir a evidência necessária sobre a eficácia da laserterapia no tratamento da $\mathrm{PB}$, todos os estudos, incluindo aqueles com bons níveis de evidência constataram que tal método pode ser válido para o desempenho do tratamento, em combinação, ou não, com outras terapias. 


\section{Responsabilidades éticas}

Proteção de pessoas e animais. Os autores declaram que para esta investigação não se realizaram experiências em seres humanos e/ou animais.

Confidencialidade dos dados. Os autores declaram que não aparecem dados de pacientes neste artigo.

Direito à privacidade e consentimento escrito. Os autores declaram que não aparecem dados de pacientes neste artigo.

\section{Conflito de interesses}

Os autores declaram não haver conflito de interesses.

I D E N T I F I C A Ç Ã O O R C I D

Ludmila M Belém (iD) 0000-0002-0727-9249

Larissa Doalla de Almeida e Silva (iD) 0000-0002-8705-9288

Dhelfeson Willya Douglas-de-Oliveira (iD) 0000-0002-8628-3122

Patrícia Furtado Gonçalves (iD) 0000-0002-1782-9654

Olga Dumont Flecha (iD 0000-0002-6999-5398

\section{R E F E R Ê N C I A S}

1. Valenca MM, Valenca LP, Lima MC. Idiopathic facial paralysis (Bell's palsy): a study of 180 patients. Arq Neuropsiquiatr. 2001;59:733-9.

2. Falavigna A, Teles AR, Giustina AD, Kleber FD. Paralisia de Bell: Fisiopatologia e tratamento. Scientia Medica. 2008;18:177-83.

3. Merabet M. Abordagem terapêutica em reabilitação da paralisia facial periférica: Revisão da literatura. Universidade Fernando Pessoa. Porto 2018. Available from: http://hdl. handle.net/10284/6729

4. Viegas VN, Kreisner PE, Mariani C, Pagnoncelli RM. Laserterapia Associada ao Tratamento da Paralisia Facial de Bell. Rev Port Estomatol Med Dent Cir Maxilofac. 2006;47:43-8.

5. Moher D, Hopewell S, Schulz KF, Montori V, Gøtzsche PC, Devereaux PJ, et al. CONSORT 2010 explanation and elaboration: updated guidelines for reporting parallel group randomised trials. Int J Surg. 2012;10:28-55.

6. He S, Wang Y, Li X, Hu D. Effectiveness of laser therapy and topical desensitising agents in treating dentine hypersensitivity: a systematic review. J Oral Rehabil. 2011;38:348-58
7. Nascimento, NPG, Gonçalves, PF, Douglas-de-Oliveira, DW, Flecha, OD. Aspiration Pneumonia and oral health: a critical review of literature. Rev Bras Odontol. 2018;75:e1058.

8. Flecha OD, Douglas de Oliveira DW, Marques LS, Gonçalves PF. A commentary on randomized clinical trials: How to produce them with a good level of evidence. Perspect Clin Res. 2016;7:75-80.

9. Alayat MS, Elsodany AM, El Fiky AA. Efficacy of high and low level laser therapy in the treatment of Bell's palsy: a randomized double blind placebo-controlled trial. Lasers Med Sci. 2014;29:335-42.

10. Ordahan B, Karahan AY. Role of low-level laser therapy added to facial expression exercises in patients with idiopathic facial (Bell's) palsy. Lasers Med Sci. 2017;32:931-6.

11. Montero TF, Zayas MSH, Andino LRC, Correoso VC. Evaluación clínica y funcional de pacientes con parálisis de Bell tratados con láser. Medisan; 2015. p.1459.

12. Murakami S, Mizobuchi M, Nakashiro Y, Doi T, Hato N, Yanagihara N. Bell palsy and herpes simplex virus: identification of viral DNA in endoneurial fluid and muscle. Ann Intern Med. 1993;124:27-30.

13. Januário PdO, Cruz AT, Garcez AG, Júnior ARdP, Nicolau RA, Lima MO. Efeitos terapêuticos do biofeedback e do laser de baixa intensidade na função física e social em pacientes com paralisia facial periférica. Revista Terapia Manual. 2012:34-39.

14. Nogueira EB, Cortine AAO, Daher A, Costa LR. Higiene oral e pneumonia em crianças em Unidade de Terapia Intensiva: revisão sistemática, Rev Assoc Paul Cir Dent. 2015;69:14-9.

15. Quinn R, Cramp F. The efficacy of electrotherapy for Bell's palsy: a systematic review. Phys Ther Rev. 2003;8:151-64. [Internet] https://www.ncbi.nlm.nih.gov/books/NBK69761/

16. Ladalardo TC, Brugnera A, Takamoto M, Pinheiro ALB, Campos RAC, Garrini AEC et al. Functional and electrophysiological evaluation of the effects of laser therapy in the treatment of peripheral facial paralysis. Proc. SPIE 4249, Lasers in Dentistry VI (27 April 2001).

17. Kneebone WJ. Enhancement of nerve regeneration by therapeutic laser. Practical Pain Management. 2010;10:70-2.

18. Rochkind S. International Conference of the World Association of Laser Therapy Sun City, North West Province, South Africa, 2008;19-22.

19. Barbosa RI, Marcolino AM, de Jesus Guirro RR, Mazzer N, Barbieri CH, de Cássia Registro Fonseca M. Comparative effects of wavelengths of low-power laser in regeneration of sciatic nerve in rats following crushing lesion. Lasers Med Sci. 2010;25:423-30.

20. Viliani T, Ricci E, Mangone G, Graziani C, Pasquetti P. Effects of Hilterapia ${ }^{\circledR}$ vs. Viscosupplementation in knee osteoarthritis patients: a randomized controlled clinical trial. Energy for Health. 2009;3:14-7.

21. Polit DF, Gillespie BM. Intention-to-treat in randomized controlled trials: recommendations for a total trial strategy. Res Nurs Health. 2010;33:355-68. 